\title{
Anion Gap Was Associated with Inhospital Mortality and Adverse Clinical Outcomes of Coronary Care Unit Patients
}

\author{
Tienan Sun $\mathbb{D}^{1},{ }^{1}$ Chenghui Cai $\mathbb{D}^{1},{ }^{1}$ Hua Shen $\left(\mathbb{D},{ }^{1}\right.$ Jiaqi Yang, ${ }^{2}$ Qianyun Guo, ${ }^{1}$ Jingrui Zhang, \\ Biyang Zhang $\mathbb{D}^{1}{ }^{1}$ Yaodong Ding $\mathbb{D}^{1},{ }^{1}$ and Yujie Zhou $\mathbb{D}^{1}$ \\ ${ }^{1}$ Beijing Anzhen Hospital Affiliated to Capital Medical University, Beijing, China \\ ${ }^{2}$ Beijing Institute of Heart, Lung and Blood Vessel Disease, Beijing, China \\ Correspondence should be addressed to Yujie Zhou; azzyj12@163.com
}

Received 25 June 2020; Revised 18 August 2020; Accepted 19 August 2020; Published 28 August 2020

Academic Editor: Giuseppe Piccione

Copyright (C) 2020 Tienan Sun et al. This is an open access article distributed under the Creative Commons Attribution License, which permits unrestricted use, distribution, and reproduction in any medium, provided the original work is properly cited.

\begin{abstract}
Background. Anion gap (AG) has been proved to be associated with prognosis of many cardiovascular diseases. This study is aimed at exploring the association of AG with inhospital all-cause mortality and adverse clinical outcomes in coronary care unit (CCU) patients. Method. All data of this study was extracted from Medical Information Mart for Intensive Care III (MIMIC-III, version 1.4) database. All patients were divided into four groups according to AG quartiles. Primary outcome was inhospital all-cause mortality. Lowess smoothing curve was drawn to describe the overall trend of inhospital mortality. Binary logistic regression analysis was performed to determine the independent effect of AG on inhospital mortality. Result. A total of 3593 patients were enrolled in this study. In unadjusted model, as AG quartiles increased, inhospital mortality increased significantly, OR increased stepwise from quartile $2(\mathrm{OR}, 95 \% \mathrm{CI}: 1.01,0.74-1.38, P=0.958)$ to quartile 4 (OR, 95\% CI: 2.72, 2.08-3.55, $P<0.001)$. After adjusting for possible confounding variables, this association was attenuated, but still remained statistically significant (quartile 1 vs. quartile 4: OR, 95\% CI: $1.02,0.72-1.45$ vs. $1.49,1.07-2.09, P=0.019)$. Moreover, CCU mortality $(P<0.001)$ and rate of acute kidney injury $(P<0.001)$ were proved to be higher in the highest AG quartiles. Length of CCU $(P<0.001)$ and hospital stay $(P<0.001)$ prolonged significantly in higher AG quartiles. Maximum sequential organ failure assessment score (SOFA) $(P<0.001)$ and simplified acute physiology score II (SAPSII) $(P<0.001)$ increased significantly as AG quartiles increased. Moderate predictive ability of AG on inhospital (AUC: 0.6291), CCU mortality (AUC: 0.6355), and acute kidney injury (AUC: 0.6096) was confirmed. The interactions were proved to be significant in hypercholesterolemia, congestive heart failure, chronic lung disease, respiratory failure, oral anticoagulants, Beta-blocks, angiotensin-converting enzyme inhibitor (ACEI)/angiotensin receptor blocker (ARB), and vasopressin treatment subgroups. Conclusion. AG was an independent risk factor of inhospital allcause mortality and was associated with adverse clinical outcomes in CCU patients.
\end{abstract}

\section{Introduction}

Despite extraordinary progress in cardiovascular field in recent decades, cardiovascular diseases still remain the major cause of death all over the world, causing about 17.5 million deaths each year $[1,2]$. Originating in 1962, coronary care unit (CCU) focuses on the treatment of patients with severe cardiovascular diseases, which greatly reduces the mortality rate of patients [3-7]. A cheap and readily available clinical indicator for assessing prognosis still makes sense for CCU patients.

As a traditional clinical indicator used to evaluate acidbase balance, anion gap (AG) has been used in clinical practice for more than 50 years $[8,9]$. AG has been proved to be associated with prognosis of many cardiovascular diseases [10-14]. A meta-analysis proved that AG was strongly related to mortality in critically ill patients [15]. Moreover, AG was confirmed to be associated with higher blood pressure [16], insulin resistance [17], and cardiorespiratory fitness [18]. In general population, higher AG was also proved to be related to cardiovascular mortality [19]. On the basis of these evidence, we hypothesized that AG could influence the prognosis of CCU patients. The purpose of this study was to explore the relationship between AG and outcomes of CCU patients. 


\section{Method}

2.1. Data Source. We retrieved all data from an openly available critical care database named Medical Information Mart for Intensive Care III (MIMIC-III, version 1.4) [20], which included more than 60000 intensive care unit (ICU) stays and more than 50000 stays for adult patients. The data in MIMIC-III were collected from June 2001 to October 2012 in Beth Israel Deaconess Medical Center, including general information (patient demographics, birth and death, ICU admission, and discharge information), vital signs, laboratory data, the balance of body fluid, reports, medication, and nursing record. Protecting Human Research Participant exam was passed to gain access to MIMIC-III database, and our certificate number is 9027152. Structured Query Language (SQL) was used to extract all patient information from MIMIC-III database.

2.2. Study Population. All adult patients ( $\geq 18$ years) admitted to CCU from MIMIC-III database were included. And only the first admission of each patient was included. Patients meeting the following criteria were excluded: (1) patients were under 18 years old, (2) length of CCU stay $<2$ days, (3) anion gap data missing, and (4) individual data missing $\geq 5 \%$. A total of 3593 patients were included in this study (Figure 1).

2.3. Data Collection. All data used in this study was extracted using SQL from MIMIC-III database. Demographics, diagnoses of heart diseases, comorbidities and medical history, laboratory parameters, medication use, and survival data were collected. Demographic data included age, gender, and race. Diagnoses of heart diseases included coronary artery disease, acute myocardial infarction, third-degree atrioventricular block, atrial fibrillation, congestive heart failure, ventricular arrhythmias (ventricular tachycardia, ventricular flutter, and ventricular fibrillation), primary cardiomyopathy (hypertrophic obstructive cardiomyopathy and other primary cardiomyopathies), valve diseases (disorders of mitral, aortic, pulmonary, and tricuspid valve; rheumatic diseases of valves and congenital diseases of valves), endocarditis, and cardiogenic shock. Cardiogenic shock was identified by the presence of appropriate International Classification of Diseases, Ninth Versions (ICD-9) diagnosis and procedure code, which was adopted by the World Health Organization to code diagnoses, and previous study confirmed the validity of ICD-9 code in recording clinical conditions in dually coded database [21]. The ICD-9 code of cardiogenic shock used in this study was 78551. Comorbidities and medical history included hypertension, diabetes, chronic liver disease, hypercholesterolemia, chronic lung disease, chronic kidney disease, malignancy, autoimmune diseases, respiratory failure, prior myocardial infarction, and prior stroke. Medication use included antiplatelet, oral anticoagulant, Beta-blocks, ACEI, ARB, statin, and vasopressin. Laboratory parameters included AG, white blood cell, platelet, hemoglobin, creatinine, blood nitrogen urea, sodium, potassium, and glucose. All laboratory parameters were extracted within 24 hours after admission to CCU.

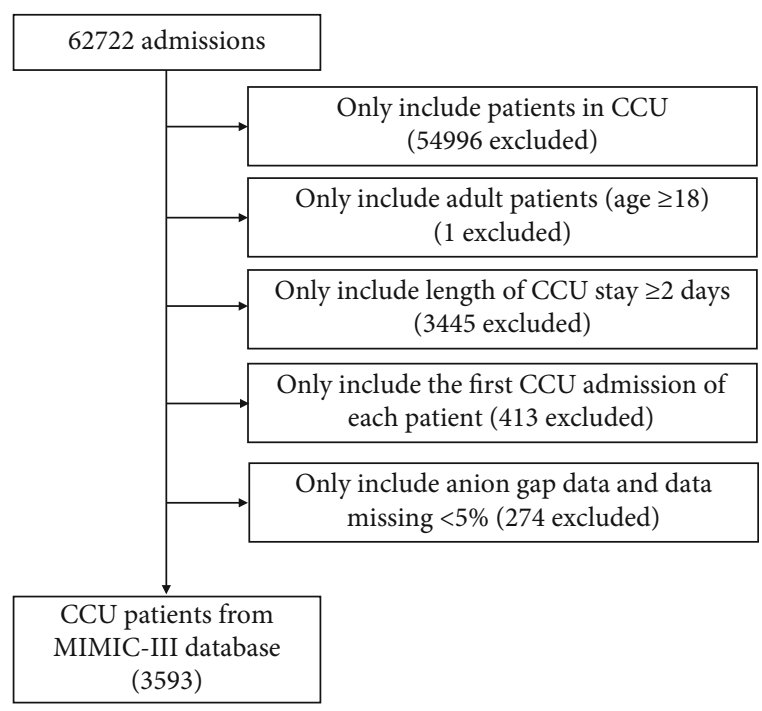

Figure 1: Flowchart of study population. CCU: coronary care unit.

AG was calculated by the following formula: $\left[\mathrm{AG}=\mathrm{Na}^{+}\right.$ $\left.(\mathrm{mmol} / \mathrm{L})+\mathrm{K}^{+}(\mathrm{mmol} / \mathrm{L})\right]-\left[\mathrm{Cl}^{-}(\mathrm{mmol} / \mathrm{L})+\mathrm{HCO}^{-}(\mathrm{mmol}\right.$ $/ \mathrm{L})$, which was generally acknowledged [22]. And AG was recorded as initial $A G$ and maximum $A G$, initial $A G$ was the first test value after admission to CCU, and maximum AG was the maximum value during CCU stay.

2.4. Outcomes. The primary outcome was inhospital all-cause mortality, secondary outcomes included CCU all-cause mortality, acute kidney injury, maximum SOFA [23], maximum SAPSII [24], length of CCU, and hospital stay. Kidney Disease: Improving Global Outcomes (KDIGO) definition [25] was used for diagnosis of acute kidney injury.

Survival information was extracted from table named "patients" of MIMIC-III database. Data of length of CCU stay was extracted from table named "icustays" of MIMICIII database. Data of length of hospital stay was extracted from table named "admissions" of MIMIC-III database. Acute kidney injury was confirmed based on KDIGO definition from table named "kdigo_creat" and "kdigo_uo" of MIMICIII database. SOFA was extracted from table named "sofa" of MIMIC-III database. SAPSII was extracted from table named "sapsii" of MIMIC-III database.

2.5. Statistical Analysis. All the patients were stratified by AG quartiles. Continuous variables were summarized as mean \pm standard deviation (SD) and median [interquartile range (IQR)]. Kruskal-Wallis test or one-way ANOVA analysis was used to test for difference. Categorical variables were summarized as number (percentage) and compared between groups using Chi-square test.

Binary logistic regression analysis was applied to identify the association between AG and inhospital all-cause mortality, and results were summarized as odds ratio (OR) with 95\% confidence interval (CI). Covariates were incorporated into regression models based on statistical evidence and clinical judgment. Local weighted regression (Lowess) was applied to fit out curves in line with overall trend. Relative 
TABLE 1: Characteristics of patients stratified by AG quartiles.

\begin{tabular}{|c|c|c|c|c|c|c|}
\hline \multirow[b]{2}{*}{ Characteristics } & \multirow[b]{2}{*}{$\begin{array}{c}\text { Total } \\
(n=3593)\end{array}$} & \multicolumn{4}{|c|}{ Quartiles of AG $(\mathrm{mmol} / \mathrm{L})$} & \multirow[b]{2}{*}{$\begin{array}{c}P \\
\text { value }\end{array}$} \\
\hline & & $\begin{array}{c}\text { Quartile } 1(n=868) \\
\text { AG }<13\end{array}$ & $\begin{array}{c}\text { Quartile } 2(n=902) \\
13 \leq \mathrm{AG}<15\end{array}$ & $\begin{array}{c}\text { Quartile } 3(n=779) \\
15 \leq \mathrm{AG}<17\end{array}$ & $\begin{array}{c}\text { Quartile } 4(n=1044) \\
15 \leq \mathrm{AG}<17\end{array}$ & \\
\hline Age (years) & $69.2 \pm 15.0$ & $69.6 \pm 14.4$ & $68.7 \pm 15.3$ & $69.4 \pm 15.0$ & $69.0 \pm 15.1$ & 0.679 \\
\hline Gender, $n(\%)$ & & & & & & 0.986 \\
\hline Male & $2042(56.8)$ & $497(57.3)$ & $510(56.5)$ & $440(56.5)$ & $595(57.0)$ & \\
\hline Female & $1551(43.2)$ & $371(42.7)$ & $392(43.5)$ & $339(43.5)$ & $449(43.0)$ & \\
\hline Race, $n(\%)$ & & & & & & 0.198 \\
\hline White & $2551(71.0)$ & $640(73.7)$ & $641(71.1)$ & $554(71.1)$ & $716(68.6)$ & \\
\hline Black & $263(7.3)$ & $52(6.0)$ & $66(7.3)$ & $53(6.8)$ & $92(8.8)$ & \\
\hline Other & $779(21.7)$ & $176(20.3)$ & $195(21.6)$ & $172(20.1)$ & $236(22.6)$ & \\
\hline Body mass index $\left(\mathrm{kg} / \mathrm{m}^{2}\right)$ & $28.2 \pm 6.9$ & $27.8 \pm 6.7$ & $28.3 \pm 7.0$ & $28.4 \pm 6.8$ & $28.3 \pm 7.2$ & 0.416 \\
\hline \multicolumn{7}{|c|}{ Diagnoses of heart diseases, $n(\%)$} \\
\hline Coronary artery disease & $1793(49.9)$ & $405(46.7)$ & $473(52.4)$ & $418(53.7)$ & $497(47.6)$ & 0.006 \\
\hline $\begin{array}{l}\text { Acute myocardial } \\
\text { infarction }\end{array}$ & $674(18.8)$ & $143(16.5)$ & $160(17.7)$ & $161(20.7)$ & $210(20.1)$ & 0.082 \\
\hline Atrial fibrillation & $1349(37.6)$ & $319(36.8)$ & $334(37.0)$ & $304(39.0)$ & $392(37.6)$ & 0.786 \\
\hline Ventricular arrhythmias & $206(5.7)$ & $31(3.6)$ & $39(4.3)$ & $47(6.0)$ & $89(8.5)$ & $<0.001$ \\
\hline $\begin{array}{l}\text { Third-degree } \\
\text { atrioventricular block }\end{array}$ & $153(4.3)$ & $34(3.9)$ & $36(4.0)$ & $37(4.8)$ & $46(4.4)$ & 0.820 \\
\hline Congestive heart failure & $1935(53.9)$ & $421(48.5)$ & $461(51.1)$ & $415(53.3)$ & $638(61.1)$ & $<0.001$ \\
\hline Primary cardiomyopathy & $294(8.2)$ & $60(6.9)$ & $62(6.9)$ & $71(9.1)$ & $101(9.7)$ & 0.048 \\
\hline Valve disease & $776(21.6)$ & $165(19.0)$ & $201(22.3)$ & $173(22.2)$ & $237(22.7)$ & 0.203 \\
\hline Endocarditis & $64(1.8)$ & $18(2.1)$ & $17(1.9)$ & $13(1.7)$ & $16(1.5)$ & 0.824 \\
\hline Cardiogenic shock & $471(13.1)$ & $70(8.1)$ & $92(10.2)$ & $94(12.1)$ & $215(20.6)$ & $<0.001$ \\
\hline \multicolumn{7}{|c|}{ Comorbidities and medical history, $n(\%)$} \\
\hline Hypertension & $1456(40.5)$ & $411(47.4)$ & $409(45.3)$ & $312(40.1)$ & $324(31.0)$ & $<0.001$ \\
\hline Diabetes & $1205(33.5)$ & $226(26.0)$ & $287(31.8)$ & $263(33.8)$ & $429(41.1)$ & $<0.001$ \\
\hline Hypercholesterolemia & $1200(33.4)$ & $313(36.1)$ & $314(34.8)$ & $271(34.8)$ & $302(28.9)$ & 0.003 \\
\hline Chronic lung disease & $885(24.6)$ & $244(28.1)$ & $195(21.6)$ & $198(25.4)$ & $248(23.8)$ & 0.013 \\
\hline Respiratory failure & $1272(35.4)$ & $279(32.1)$ & $280(31.0)$ & $264(33.9)$ & $449(43.0)$ & $<0.001$ \\
\hline Chronic kidney disease & $752(20.9)$ & $106(12.2)$ & $165(18.3)$ & $171(22.0)$ & $310(29.7)$ & $<0.001$ \\
\hline Chronic liver disease & $125(3.5)$ & $39(4.5)$ & $22(2.4)$ & $19(2.4)$ & $45(4.3)$ & 0.017 \\
\hline Malignancy & $518(14.4)$ & $125(14.4)$ & $158(17.5)$ & $105(13.5)$ & $130(12.5)$ & 0.013 \\
\hline Autoimmune disease & $156(4.3)$ & $40(4.6)$ & $35(3.9)$ & $34(4.4)$ & $47(4.5)$ & 0.879 \\
\hline $\begin{array}{l}\text { Prior myocardial } \\
\text { infarction }\end{array}$ & $323(9.0)$ & $77(8.9)$ & $80(8.9)$ & $83(10.7)$ & $83(8.0)$ & 0.256 \\
\hline Prior stroke & $85(2.4)$ & $19(2.2)$ & $24(2.7)$ & $12(1.5)$ & $30(2.9)$ & 0.270 \\
\hline \multicolumn{7}{|l|}{ Laboratory parameters } \\
\hline $\mathrm{AG}(\mathrm{mmol} / \mathrm{L})$ & $15.0 \pm 3.6$ & $10.9 \pm 1.2$ & $13.5 \pm 0.5$ & $15.5 \pm 0.5$ & $19.4 \pm 2.6$ & $<0.001$ \\
\hline Maximum AG & $17.7 \pm 4.3$ & $14.7 \pm 3.4$ & $16.2 \pm 2.9$ & $17.7 \pm 3.0$ & $21.4 \pm 4.1$ & $<0.001$ \\
\hline White blood cell $\left(10^{9} / \mathrm{L}\right)$ & $11.7 \pm 5.6$ & $10.0 \pm 4.6$ & $10.8 \pm 4.8$ & $11.7 \pm 5.4$ & $13.7 \pm 6.3$ & $<0.001$ \\
\hline Platelet $\left(10^{9} / \mathrm{L}\right)$ & $236.8 \pm 96.8$ & $220.1 \pm 91.3$ & $234.8 \pm 93.6$ & $242.0 \pm 95.1$ & $248.4 \pm 102.9$ & $<0.001$ \\
\hline Hemoglobin (g/dL) & $11.5 \pm 2.0$ & $11.2 \pm 1.8$ & $11.4 \pm 1.9$ & $11.6 \pm 2.0$ & $11.6 \pm 2.1$ & $<0.001$ \\
\hline Glucose (mg/dL) & $154.6 \pm 75.2$ & $132.1 \pm 52.0$ & $141.5 \pm 56.9$ & $154.6 \pm 69.0$ & $184.6 \pm 97.0$ & $<0.001$ \\
\hline Creatinine $(\mathrm{mg} / \mathrm{dL})$ & $1.58 \pm 1.40$ & $1.06 \pm 0.63$ & $1.24 \pm 0.79$ & $1.45 \pm 0.95$ & $2.41 \pm 2.04$ & $<0.001$ \\
\hline $\begin{array}{l}\text { Blood nitrogen urea } \\
(\mathrm{mg} / \mathrm{dL})\end{array}$ & $31.0 \pm 21.6$ & $22.6 \pm 14.1$ & $25.7 \pm 16.2$ & $31.3 \pm 20.7$ & $42.4 \pm 26.2$ & $<0.001$ \\
\hline Sodium (mmol/L) & $138.0 \pm 4.6$ & $138.4 \pm 4.3$ & $138.3 \pm 4.2$ & $138.2 \pm 4.2$ & $137.2 \pm 5.3$ & $<0.001$ \\
\hline
\end{tabular}


TABLE 1: Continued.

\begin{tabular}{|c|c|c|c|c|c|c|}
\hline \multirow[b]{2}{*}{ Characteristics } & \multirow[b]{2}{*}{$\begin{array}{c}\text { Total } \\
(n=3593)\end{array}$} & \multicolumn{4}{|c|}{ Quartiles of $A G(\mathrm{mmol} / \mathrm{L})$} & \multirow[b]{2}{*}{$\begin{array}{c}P \\
\text { value }\end{array}$} \\
\hline & & $\begin{array}{c}\text { Quartile } 1(n=868) \\
\text { AG }<13\end{array}$ & $\begin{array}{c}\text { Quartile } 2(n=902) \\
13 \leq \mathrm{AG}<15\end{array}$ & $\begin{array}{c}\text { Quartile } 3(n=779) \\
15 \leq \mathrm{AG}<17\end{array}$ & $\begin{array}{c}\text { Quartile } 4(n=1044) \\
15 \leq \mathrm{AG}<17\end{array}$ & \\
\hline Potassium $(\mathrm{mmol} / \mathrm{L})$ & $4.2 \pm 0.8$ & $4.0 \pm 0.6$ & $4.1 \pm 0.7$ & $4.2 \pm 0.7$ & $4.4 \pm 0.9$ & $<0.001$ \\
\hline \multicolumn{7}{|l|}{ Medication use, $n(\%)$} \\
\hline Antiplatelet & $2274(63.3)$ & $556(64.1)$ & $587(65.9)$ & $480(61.6)$ & $651(62.4)$ & 0.425 \\
\hline Oral anticoagulants & $1047(29.1)$ & $259(29.8)$ & $276(30.6)$ & $232(29.8)$ & $280(26.8)$ & 0.260 \\
\hline Beta-blocks & $2513(69.9)$ & 624 (71.9) & $644(71.4)$ & $533(68.4)$ & $712(68.2)$ & 0.184 \\
\hline ACEI/ARB & $1882(52.4)$ & $475(54.7)$ & $495(54.9)$ & $422(54.2)$ & $490(46.9)$ & 0.001 \\
\hline Statin & 2095 (58.3) & $512(59.0)$ & $552(61.2)$ & $458(58.8)$ & $573(54.9)$ & 0.039 \\
\hline Vasopressin & $234(6.5)$ & $38(4.4)$ & $46(5.1)$ & $45(5.8)$ & $105(10.1)$ & $<0.001$ \\
\hline
\end{tabular}

Continuous variables were presented as mean $\pm \mathrm{SD}$. Categorical variables were presented as number (percentage). Abbreviation: AG: anion gap; ACEI: angiotensin-converting enzyme inhibitor; ARB: angiotensin receptor blocker.

TABLe 2: Outcomes of patients stratified by AG quartiles.

\begin{tabular}{|c|c|c|c|c|c|c|}
\hline \multirow[b]{2}{*}{ Outcomes } & \multirow[b]{2}{*}{$\begin{array}{c}\text { Total } \\
(n=3593)\end{array}$} & \multicolumn{4}{|c|}{ Quartiles of $A G(\mathrm{mmol} / \mathrm{L})$} & \multirow{2}{*}{$\begin{array}{c}P \\
\text { value }\end{array}$} \\
\hline & & $\begin{array}{c}\text { Quartile } 1(n=868) \\
\text { AG }<13\end{array}$ & $\begin{array}{c}\text { Quartile } 2(n=902) \\
13 \leq \mathrm{AG}<15\end{array}$ & $\begin{array}{c}\text { Quartile } 3(n=779) \\
15 \leq \mathrm{AG}<17\end{array}$ & $\begin{array}{c}\text { Quartile } 4(n=1044) \\
15 \leq \mathrm{AG}<17\end{array}$ & \\
\hline Inhospital mortality, $n(\%)$ & $515(14.3)$ & $85(9.8)$ & $89(9.9)$ & $103(13.2)$ & $238(22.8)$ & $<0.001$ \\
\hline CCU mortality, $n(\%)$ & $399(11.1)$ & $55(6.3)$ & $73(8.1)$ & $88(11.3)$ & $183(17.5)$ & $<0.001$ \\
\hline Length of CCU stay (days) & $\begin{array}{c}3.9(2.8- \\
6.6)\end{array}$ & $3.5(2.6-5.9)$ & $3.7(2.8-5.9)$ & $3.9(2.7-6.7)$ & $4.4(3.0-7.3)$ & $<0.001$ \\
\hline $\begin{array}{l}\text { Length of hospital stay } \\
\text { (days) }\end{array}$ & $\begin{array}{c}8.3(5.3- \\
13.9)\end{array}$ & $7.3(4.8-12.5)$ & $7.8(5.0-13.2)$ & $8.5(5.3-13.7)$ & $9.7(4.0-15.7)$ & $<0.001$ \\
\hline Acute kidney injury, $n(\%)$ & $1924(53.6)$ & $371(42.7)$ & $434(48.1)$ & $429(55.1)$ & $690(66.1)$ & $<0.001$ \\
\hline Maximum SOFA & $4(2-6)$ & $3(1-5)$ & $3(2-5)$ & $4(2-6)$ & $5(3-8)$ & $<0.001$ \\
\hline Maximum SAPSII & $36(28-46)$ & $33(27-40)$ & $34(26-43)$ & $36(28-44)$ & $42(33-52)$ & $<0.001$ \\
\hline
\end{tabular}

Nonnormally distributed continuous variables were presented as median (IQR). Categorical variables were presented as number (percentage). Abbreviation: AG: anion gap; CCU: coronary care unit; SOFA: sequential organ failure assessment score; SAPS II: simplified acute physiology score II.

operating characteristic (ROC) curves were used to evaluate predictive ability of AG on inhospital all-cause mortality. All data processing and analysis were performed by Stata V.11.2. All tests were two sided, and $P<0.05$ was considered statistically significant.

\section{Result}

3.1. Patient Characteristics. After screening step by step, a total of 3593 patients admitted to CCU were extracted from MIMIC-III database (Figure 1), most of whom were white and male. The baseline characteristics of patients stratified by $A G$ quartiles are presented in Table 1. Initial AG and maximum AG of all patients were $15.0 \pm 3.6 \mathrm{mmol} / \mathrm{L}$ and $17.7 \pm 4.3 \mathrm{mmol} / \mathrm{L}$, respectively. As AG quartiles increased, rates of ventricular arrhythmias, congestive heart failure, primary cardiomyopathy, cardiogenic shock, diabetes, respiratory failure, and chronic kidney diseases increased. But rates of coronary artery disease, hypertension, hypercholesterolemia, chronic lung diseases, and malignancy decreased as AG quartiles increased. Moreover, patients with higher AG had higher white blood cell, platelet, hemoglobin, glucose, creatinine, blood nitrogen urea, and potassium. Patients with higher AG also received less ACEI/ARB and statin treatment but more vasopressin treatment.

3.2. Outcomes. The primary outcome was inhospital all-cause mortality. As shown in Table 2, the inhospital mortality of all patients in this study was $14.3 \%$. As AG quartiles increased, inhospital mortality increased gradually (quartile 1 vs. quartile $4: 9.8 \%$ vs. $22.8 \%, P<0.001$ ); the same conclusion was drawn by Lowess smoothing curve shown in Figure 2. From unadjusted model comparing inhospital all-cause mortality among different AG groups, we observed that as AG quartiles increased, inhospital mortality increased significantly, OR increased stepwise from quartile 2 (OR, 95\% CI: 1.01, 0.74$1.38, P=0.958)$ to quartile 4 (OR, 95\% CI: $2.72,2.08-3.55$, $P<0.001)$. After adjusting for more variables in model 3 , this association was weakened, but still remained statistically significant (quartile 1 vs. quartile 4: OR, 95\% CI: 1.02, 0.72-1.45 vs. $1.49,1.07-2.09, P=0.019$ ) (Table 3).

Secondary outcomes were CCU mortality, acute kidney injury, maximum SOFA, maximum SAPSII, length of CCU, and hospital stay. As shown in Table 2, CCU mortality of all patients was $11.1 \%$, and as AG quartiles increased, the rate of CCU mortality increased stepwise from quartile $1(6.3 \%)$ 


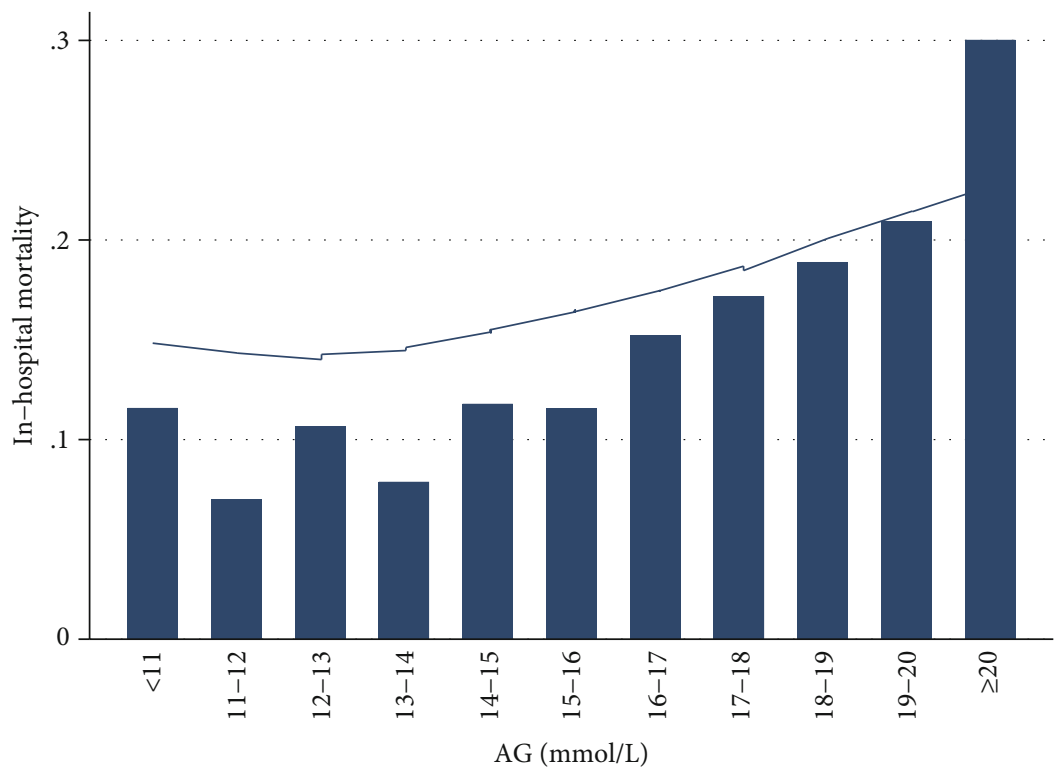

FIGURE 2: Association between anion gap and inhospital all-cause mortality presented through Lowess smoothing. Abbreviation: AG: anion gap.

TABLE 3: The association between AG and inhospital all-cause mortality.

\begin{tabular}{lccc}
\hline & \multicolumn{3}{c}{ AG $(\mathrm{mmol})$} \\
& OR $(95 \% \mathrm{CI})$ & $P$ value & $P$ for trend \\
\hline Model 1 & & & $<0.001$ \\
Quartile 1: AG $<13$ & Ref & & \\
Quartile 2: $13 \leq \mathrm{AG}<15$ & $1.01(0.74-1.38)$ & 0.958 & \\
Quartile 3: $15 \leq \mathrm{AG}<17$ & $1.40(1.03-1.90)$ & 0.029 & \\
Quartile 4: AG $\geq 17$ & $2.72(2.08-3.55)$ & $<0.001$ & \\
Continuous & $1.13(1.10-1.16)$ & $<0.001$ & \\
Model 2 & & & $<0.001$ \\
Quartile 1: AG $<13$ & Ref & & \\
Quartile 2: $13 \leq \mathrm{AG}<15$ & $1.02(0.75-1.40)$ & 0.891 & \\
Quartile 3: $15 \leq \mathrm{AG}<17$ & $1.40(1.03-1.91)$ & 0.031 & \\
Quartile 4: AG $\geq 17$ & $2.78(2.12-3.63)$ & $<0.001$ & \\
Continuous & $1.14(1.11-1.16)$ & $<0.001$ & \\
Model 3 & & & \\
Quartile 1: AG $<13$ & Ref & & \\
Quartile 2: $13 \leq \mathrm{AG}<15$ & $1.02(0.72-1.45)$ & 0.897 & \\
Quartile 3: $15 \leq \mathrm{AG}<17$ & $1.22(0.86-1.73)$ & 0.258 & \\
Quartile 4: $\mathrm{AG} \geq 17$ & $1.49(1.07-2.09)$ & 0.019 & \\
Continuous & $1.06(1.02-1.09)$ & 0.001 & \\
\hline
\end{tabular}

Models were derived from binary logistic regression analysis. Model 1: unadjusted. Model 2: adjusted for age, gender, and race. Model 3: adjusted for age, gender, race, body mass index, coronary heart disease, acute myocardial infarction, atrial fibrillation, ventricular arrhythmias, third-degree atrioventricular block, congestive heart failure, primary cardiomyopathy, valve disease, endocarditis, cardiogenic shock, hypertension, diabetes, hypercholesterolemia, respiratory failure, chronic kidney disease, chronic liver disease, chronic lung disease, malignancy, autoimmune disease, prior myocardial infarction, prior stroke, oral anticoagulants, statin, vasopressin, angiotensin-converting enzyme inhibitor, angiotensin receptor blocker, antiplatelet, blood nitrogen urea, white blood cell, sodium, and creatinine. Abbreviation: AG: anion gap; OR: odds ratio; CI: confidence interval. to quartile $4(17.5 \%)(P<0.001)$. Length of CCU stay (quartile 1 vs. quartile $4: 3.5(2.6-5.9)$ vs. $4.4(3.0-7.3), P<0.001)$ and length of hospital staying (quartile 1 vs. quartile $4: 7.3$ (4.8-12.5) vs. 9.7 (4.0-15.7), $P<0.001)$ increased significantly as AG increased. A total of 1924 patients were diagnosed with acute kidney injury based on KDIGO definition, and the incidence of acute kidney injury increased gradually from quartile $1(42.7 \%)$ to quartile $4(66.1 \%)(P<0.001)$. Moreover, patients in highest AG quartile had highest maximum SAPSII (quartile 1 vs. quartile 4: 33 (27-40) vs. 42 (33-52), $P<0.001$ ) and highest maximum SOFA (quartile 1 vs. quartile $4: 3$ (1-5) vs. 5 (3-8), $P<0.001)$.

The relationship between inhospital mortality and AG quartiles in different subgroups is shown in Table 4 . We did not observe significant interactions in most subgroups. Patients with chronic lung disease, congestive heart failure, and respiratory failure had lower risk of inhospital death. Moreover, patients who received oral anticoagulants, Betablocks, ACEI/ARB, and vasopressin treatment had lower risk of inhospital death too. But patients with hypercholesterolemia had higher risk of inhospital death.

As presented in Figure 3, moderate predictive ability of AG on inhospital (AUC, 95\% CI: 0.6291, 0.6019-0.6564), CCU mortality (AUC, 95\% CI: 0.6355, 0.6060-0.6650), and acute kidney injury (AUC, 95\% CI: 0.6096, 0.5914-0.6278) was confirmed.

\section{Discussion}

This study explored the association of AG with inhospital mortality and other adverse outcomes of CCU patients. The main findings were as follows: (1) as AG quartiles increased, inhospital all-cause mortality increased significantly. (2) As AG quartiles increased, CCU mortality and the rate of acute kidney injury increased. (3) Patients with higher AG had higher maximum SOFA and maximum SAPSII. (4) Length of CCU and hospital stay prolonged significantly in higher 
TABLE 4: Subgroup analysis of associations between inhospital all-cause mortality and AG (mmol/L).

\begin{tabular}{|c|c|c|c|c|c|c|}
\hline Subgroups & $n$ & $\begin{array}{c}\text { Quartile } 1 \\
\text { AG }<13\end{array}$ & $\begin{array}{c}\text { Quartile } 2 \\
13 \leq \mathrm{AG}<15\end{array}$ & $\begin{array}{c}\text { Quartile } 3 \\
15 \leq \mathrm{AG}<17\end{array}$ & $\begin{array}{c}\text { Quartile } 4 \\
15 \leq \mathrm{AG}<17\end{array}$ & $\begin{array}{c}P \\
\text { for interaction }\end{array}$ \\
\hline Gender & & & & & & 0.839 \\
\hline Male & 2042 & Ref & $1.21(0.80-1.84)$ & $1.32(0.86-2.01)$ & $2.98(2.07-4.27)$ & \\
\hline Female & 1551 & Ref & $0.79(0.49-1.27)$ & $1.50(0.96-2.33)$ & $2.43(1.64-3.61)$ & \\
\hline Age (years) & & & & & & 0.968 \\
\hline$<72$ & 1832 & Ref & $1.08(0.67-1.75)$ & $1.09(0.66-1.80)$ & $2.82(1.87-4.25)$ & \\
\hline$\geq 72$ & 1761 & Ref & $0.97(0.64-1.47)$ & $1.62(1.10-2.39)$ & $2.71(1.90-3.85)$ & \\
\hline Race & & & & & & 0.244 \\
\hline White & 2551 & Ref & $1.07(0.74-1.54)$ & $1.30(0.91-1.87)$ & $2.57(1.88-3.52)$ & \\
\hline Black & 263 & Ref & $0.14(0.16-1.28)$ & $0.77(0.19-3.03)$ & $2.29(0.80-6.57)$ & \\
\hline Other & 779 & Ref & $1.13(0.57-2.22)$ & $1.98(1.05-3.73)$ & $3.41(1.91-6.07)$ & \\
\hline Body mass index $\left(\mathrm{kg} / \mathrm{m}^{2}\right)$ & & & & & & 0.332 \\
\hline$<27$ & 1792 & Ref & $0.96(0.64-1.46)$ & $1.49(0.99-2.22)$ & $2.45(1.72-3.48)$ & \\
\hline$\geq 27$ & 1801 & Ref & $1.08(0.67-1.75)$ & $1.35(0.84-2.17)$ & $3.14(2.09-4.72)$ & \\
\hline Coronary artery disease & & & & & & 0.530 \\
\hline Yes & 1793 & Ref & $1.20(0.73-1.97)$ & $1.68(1.04-2.73)$ & $3.10(2.00-4.81)$ & \\
\hline No & 1800 & Ref & $0.94(0.62-1.41)$ & $1.31(0.88-1.95)$ & $2.55(1.82-3.57)$ & \\
\hline Acute myocardial infarction & & & & & & 0.269 \\
\hline Yes & 674 & Ref & $1.15(0.50-2.62)$ & $2.10(0.99-4.46)$ & $3.65(1.83-7.30)$ & \\
\hline No & 2919 & Ref & $0.99(0.70-1.39)$ & $1.29(0.92-1.81)$ & $2.58(1.93-3.45)$ & \\
\hline Atrial fibrillation & & & & & & 0.921 \\
\hline Yes & 1349 & Ref & $1.16(0.72-1.86)$ & $1.40(0.88-2.24)$ & $2.91(1.93-4.40)$ & \\
\hline No & 2244 & Ref & $0.90(0.59-1.37)$ & $1.39(0.93-1.08)$ & $2.59(1.83-3.68)$ & \\
\hline Ventricular arrhythmias & & & & & & 0.065 \\
\hline Yes & 206 & Ref & $6.56(0.76-56.55)$ & $9.17(1.12-75.13)$ & $17.68(2.30-135.7)$ & \\
\hline No & 3387 & Ref & $0.94(0.68-1.30)$ & $1.29(0.94-1.76)$ & $2.45(1.86-3.22)$ & \\
\hline Third-degree atrioventricular block & & & & & & 0.687 \\
\hline Yes & 153 & Ref & $0.30(0.03-2.99)$ & $0.91(0.17-4.86)$ & $2.51(0.63-10.1)$ & \\
\hline No & 3440 & Ref & $1.04(0.76-1.42)$ & $1.43(1.05-1.95)$ & $2.73(2.08-3.58)$ & \\
\hline Congestive heart failure & & & & & & 0.013 \\
\hline Yes & 1935 & Ref & $0.84(0.56-1.27)$ & $1.15(0.77-1.71)$ & $1.97(1.40-2.78)$ & \\
\hline No & 1658 & Ref & $1.26(0.77-2.05)$ & $1.78(1.11-2.88)$ & $4.07(2.66-6.23)$ & \\
\hline Primary cardiomyopathy & & & & & & 0.994 \\
\hline Yes & 294 & Ref & $0.97(0.23-4.05)$ & $1.78(0.51-6.22)$ & $2.64(0.84-8.29)$ & \\
\hline No & 3299 & Ref & $1.01(0.73-1.39)$ & $1.39(1.01-1.91)$ & $2.76(2.10-3.64)$ & \\
\hline Valve disease & & & & & & 0.919 \\
\hline Yes & 776 & Ref & $0.75(0.35-1.60)$ & $1.38(0.69-2.78)$ & $2.34(1.264 .37)$ & \\
\hline No & 2817 & Ref & $1.08(0.77-1.53)$ & $1.42(1.01-1.99)$ & $2.84(2.12-3.82)$ & \\
\hline Endocarditis & & & & & & 0.617 \\
\hline Yes & 64 & Ref & $0.67(0.10-4.58)$ & $0.91(0.13-6.40)$ & $3.89(0.80-18.97)$ & \\
\hline No & 3529 & Ref & $1.02(0.74-1.40)$ & $1.42(1.04-1.94)$ & $2.71(2.07-3.56)$ & \\
\hline Cardiogenic shock & & & & & & 0.352 \\
\hline Yes & 471 & Ref & $1.00(0.48-2.09)$ & $1.43(0.70-2.92)$ & $1.88(1.01-3.51)$ & \\
\hline No & 3122 & Ref & $0.97(0.68-1.37)$ & $1.30(0.92-1.83)$ & $2.55(1.88-3.44)$ & \\
\hline Hypertension & & & & & & 0.126 \\
\hline Yes & 1456 & Ref & $1.01(0.60-1.67)$ & $1.50(0.90-2.48)$ & $3.44(2.21-5.38)$ & \\
\hline No & 2137 & Ref & $1.00(0.67-1.48)$ & $1.30(0.88-1.91)$ & $2.27(1.62-3.17)$ & \\
\hline Diabetes & & & & & & 0.914 \\
\hline Yes & 1205 & Ref & $1.35(0.73-2.49)$ & $1.72(0.94-3.13)$ & $2.94(1.72-5.02)$ & \\
\hline
\end{tabular}


TABle 4: Continued.

\begin{tabular}{|c|c|c|c|c|c|c|}
\hline Subgroups & $n$ & $\begin{array}{c}\text { Quartile } 1 \\
\text { AG }<13\end{array}$ & $\begin{array}{c}\text { Quartile } 2 \\
13 \leq \mathrm{AG}<15\end{array}$ & $\begin{array}{c}\text { Quartile } 3 \\
15 \leq \mathrm{AG}<17\end{array}$ & $\begin{array}{c}\text { Quartile } 4 \\
15 \leq \mathrm{AG}<17\end{array}$ & $\begin{array}{c}P \\
\text { for interaction }\end{array}$ \\
\hline No & 2388 & Ref & $0.91(0.63-1.32)$ & $1.32(0.93-1.90)$ & $2.79(2.04-3.82)$ & \\
\hline Hypercholesterolemia & & & & & & 0.019 \\
\hline Yes & 1200 & Ref & $1.54(0.80-2.95)$ & $2.22(1.18-4.19)$ & $4.89(2.75-8.69)$ & \\
\hline No & 2393 & Ref & $0.88(0.61-1.26)$ & $1.20(0.84-1.71)$ & $2.17(1.60-2.95)$ & \\
\hline Chronic lung disease & & & & & & $<0.001$ \\
\hline Yes & 885 & Ref & $0.94(0.56-1.56)$ & $0.72(0.42-1.22)$ & $1.55(0.99-2.41)$ & \\
\hline No & 2708 & Ref & $1.18(0.78-1.77)$ & $2.04(1.39-3.01)$ & $3.82(2.70-5.42)$ & \\
\hline Respiratory failure & & & & & & 0.005 \\
\hline Yes & 1272 & Ref & $0.71(0.46-1.09)$ & $1.14(0.76-1.72)$ & $1.67(1.18-2.38)$ & \\
\hline No & 2321 & Ref & $1.62(0.99-2.65)$ & $1.85(1.12-3.04)$ & $4.26(2.74-6.62)$ & \\
\hline Chronic kidney disease & & & & & & 0.813 \\
\hline Yes & 752 & Ref & $1.49(0.65-3.40)$ & $1.35(0.59-3.10)$ & $2.86(1.37-5.97)$ & \\
\hline No & 2841 & Ref & $0.93(0.66-1.31)$ & $1.45(1.04-2.01)$ & $2.78(2.08-3.73)$ & \\
\hline Chronic liver disease & & & & & & 0.538 \\
\hline Yes & 125 & Ref & $1.22(0.30-4.90)$ & $1.03(0.23-4.66)$ & $2.23(0.76-6.60)$ & \\
\hline No & 3468 & Ref & $1.02(0.74-1.40)$ & $1.44(1.05-1.97)$ & $2.76(2.10-3.63)$ & \\
\hline Malignancy & & & & & & 0.109 \\
\hline Yes & 518 & Ref & $0.97(0.49-1.94)$ & $1.14(0.55-2.39)$ & $1.82(0.95-3.52)$ & \\
\hline No & 3075 & Ref & $1.00(0.70-1.42)$ & $1.47(1.05-2.06)$ & $2.94(2.19-3.95)$ & \\
\hline Autoimmune disease & & & & & & 0.680 \\
\hline Yes & 156 & Ref & $0.53(0.12-2.30)$ & $1.21(0.35-4.18)$ & $1.94(0.65-5.77)$ & \\
\hline No & 3437 & Ref & $1.04(0.76-1.44)$ & $1.42(1.04-1.94)$ & $2.78(2.11-3.66)$ & \\
\hline Prior myocardial infarction & & & & & & 0.706 \\
\hline Yes & 323 & Ref & $1.43(0.51-3.97)$ & $1.22(0.43-3.44)$ & $2.77(1.09-7.06)$ & \\
\hline No & 3270 & Ref & $0.97(0.70-1.35)$ & $1.43(1.04-1.96)$ & $2.71(2.06-3.58)$ & \\
\hline Prior stroke & & & & & & 0.413 \\
\hline Yes & 61 & Ref & - & $3.6(0.29-44.82)$ & $3.6(0.39-33.50)$ & \\
\hline No & 3508 & Ref & $1.03(0.75-1.41)$ & $1.38(1.02-1.88)$ & $2.72(2.08-3.55)$ & \\
\hline Antiplatelet & & & & & & 0.678 \\
\hline Yes & 2274 & Ref & $1.02(0.69-1.53)$ & $1.61(1.09-2.36)$ & $2.81(2.00-3.96)$ & \\
\hline No & 1319 & Ref & $0.99(0.60-1.64)$ & $1.12(0.68-1.84)$ & $2.57(1.68-3.93)$ & \\
\hline Oral anticoagulants & & & & & & 0.011 \\
\hline Yes & 1047 & Ref & $0.99(0.51-1.89)$ & $0.57(0.26-1.25)$ & $1.57(0.86-2.86)$ & \\
\hline No & 2546 & Ref & $1.02(0.71-1.46)$ & $1.69(1.20-2.37)$ & $3.06(2.26-4.13)$ & \\
\hline Beta-blockers & & & & & & 0.002 \\
\hline Yes & 2513 & Ref & $0.90(0.61-1.31)$ & $1.24(0.85-1.80)$ & $1.98(1.43-2.76)$ & \\
\hline No & 1080 & Ref & $1.28(0.74-2.23)$ & $1.75(1.03-2.98)$ & $4.58(2.86-7.34)$ & \\
\hline ACEI/ARB & & & & & & $<0.001$ \\
\hline Yes & 1882 & Ref & $0.96(0.58-1.59)$ & $1.17(0.71-1.95)$ & $1.40(0.87-2.24)$ & \\
\hline No & 1711 & Ref & $1.04(0.70-1.56)$ & $1.56(1.06-2.31)$ & $3.43(2.45-4.81)$ & \\
\hline Statin & & & & & & 0.846 \\
\hline Yes & 2095 & Ref & $1.02(0.65-1.60)$ & $1.45(0.93-2.24)$ & $2.62(1.78-3.85)$ & \\
\hline No & 1498 & Ref & $1.02(0.66-1.59)$ & $1.37(0.89-2.10)$ & $2.75(1.90-3.98)$ & \\
\hline Vasopressin & & & & & & 0.042 \\
\hline Yes & 234 & Ref & $0.65(0.27-1.56)$ & $0.89(0.37-2.11)$ & $1.18(0.56-2.47)$ & \\
\hline No & 3359 & Ref & $1.05(0.74-1.48)$ & $1.45(1.04-2.04)$ & $2.78(2.06-3.74)$ & \\
\hline White blood cell $\left(10^{9} / \mathrm{L}\right)$ & & & & & & 0.057 \\
\hline$<10.5$ & 1778 & Ref & $0.80(0.52-1.23)$ & $1.22(0.79-1.87)$ & $1.91(1.29-2.84)$ & \\
\hline
\end{tabular}


TABLE 4: Continued.

\begin{tabular}{|c|c|c|c|c|c|c|}
\hline Subgroups & $n$ & $\begin{array}{c}\text { Quartile } 1 \\
\text { AG }<13\end{array}$ & $\begin{array}{c}\text { Quartile } 2 \\
13 \leq \mathrm{AG}<15\end{array}$ & $\begin{array}{c}\text { Quartile } 3 \\
15 \leq \mathrm{AG}<17\end{array}$ & $\begin{array}{c}\text { Quartile } 4 \\
15 \leq \mathrm{AG}<17\end{array}$ & $\begin{array}{c}P \\
\text { for interaction }\end{array}$ \\
\hline$\geq 10.5$ & 1815 & Ref & $1.25(0.78-2.01)$ & $1.54(0.98-2.43)$ & $3.10(2.07-4.65)$ & \\
\hline Platelet $\left(10^{9} / \mathrm{L}\right)$ & & & & & & 0.170 \\
\hline$<221$ & 1793 & Ref & $1.10(0.71-1.72)$ & $1.80(1.18-2.75)$ & $3.30(2.27-4.81)$ & \\
\hline$\geq 221$ & 1800 & Ref & $0.91(0.58-1.41)$ & $1.07(0.69-1.66)$ & $2.22(1.52-3.24)$ & \\
\hline Hemoglobin $(\mathrm{g} / \mathrm{dL})$ & & & & & & 0.223 \\
\hline$<11.4$ & 1777 & Ref & $0.93(0.62-1.39)$ & $1.24(0.82-1.86)$ & $2.42(1.70-3.43)$ & \\
\hline$\geq 11.4$ & 1816 & Ref & $1.16(0.71-1.90)$ & $1.73(1.09-2.76)$ & $3.31(2.17-5.04)$ & \\
\hline Glucose (mg/dL) & & & & & & 0.828 \\
\hline$<132$ & 1773 & Ref & $1.12(0.74-1.70)$ & $1.42(0.93-2.16)$ & $2.88(1.97-4.22)$ & \\
\hline$\geq 132$ & 1820 & Ref & $0.86(0.53-1.39)$ & $1.32(0.84-2.08)$ & $2.44(1.65-3.62)$ & \\
\hline Creatinine $(\mathrm{mg} / \mathrm{dL})$ & & & & & & 0.177 \\
\hline$<1.1$ & 1537 & Ref & $0.99(0.64-1.54)$ & $1.24(0.78-1.97)$ & $2.20(1.40-3.45)$ & \\
\hline$\geq 1.1$ & 2056 & Ref & $0.96(0.61-1.52)$ & $1.37(0.89-2.11)$ & $2.52(1.72-3.68)$ & \\
\hline Blood nitrogen urea $(\mathrm{mg} / \mathrm{dL})$ & & & & & & 0.365 \\
\hline$<24$ & 1728 & Ref & $1.08(0.68-1.73)$ & $1.34(0.81-2.20)$ & $2.79(1.77-4.39)$ & \\
\hline$\geq 24$ & 1865 & Ref & $0.83(0.54-1.27)$ & $1.06(0.71-1.27)$ & $1.76(1.24-2.50)$ & \\
\hline Sodium (mmol/L) & & & & & & 0.123 \\
\hline$<138$ & 1456 & Ref & $1.16(0.73-1.84)$ & $1.26(0.79-2.00)$ & $2.28(1.53-3.39)$ & \\
\hline$\geq 138$ & 2137 & Ref & $0.89(0.58-1.37)$ & $1.50(1.00-2.25)$ & $3.02(2.10-4.33)$ & \\
\hline Potassium $(\mathrm{mmol} / \mathrm{L})$ & & & & & & 0.290 \\
\hline$<4.1$ & 1625 & Ref & $0.95(0.60-1.50)$ & $1.70(1.09-2.64)$ & $3.12(2.09-4.65)$ & \\
\hline$\geq 4.1$ & 1968 & Ref & $1.04(0.68-1.61)$ & $1.17(0.77-1.79)$ & $2.39(1.66-3.44)$ & \\
\hline
\end{tabular}

Binary logistic regression analysis was used, and results were presented as OR (odds ratio) and 95\% CI (confidence interval). Abbreviation: AG: anion gap; ACEI: angiotensin-converting enzyme inhibitor; ARB: angiotensin receptor blocker.

AG quartiles. (5) Moderate predictive ability of AG on inhospital, CCU mortality, and acute kidney injury was confirmed. (6) The interactions were proved to be significant in hypercholesterolemia, congestive heart failure, chronic lung disease, respiratory failure, oral anticoagulants, Beta-blocks, ACEI/ARB, and vasopressin treatment subgroups.

Acid-base balance is very important for the maintenance of normal physiological function and cell metabolism [26]. As a common indicator to evaluate acid-base balance, AG is often used to define the types and causes of metabolic acidosis. Clinically, AG is usually calculated by the concentration of serum sodium, potassium, chloride, and bicarbonate [27], which is inexpensive and readily available.

AG has been proved to be associated with prognosis of many cardiovascular diseases [10-14]. Previous study which enrolled 18115 patients with coronary disease showed that higher AG was associated with worse cardiac function, more severe clinical symptoms, and acute myocardial infarction: for every unit increase in AG, the 30-day risk of all-cause death increased by 0.244 times [10]. In patients with myocardial infarction, increased AG was also associated with higher mortality and cardiogenic shock [12]. Another research enrolled 63 patients with cardiogenic shock following STsegment elevation myocardial infarction (STEMI) came to a similar conclusion that higher AG was associated with higher mortality [13]. For patients with STEMI, AG was proved to be an independent risk factor for high inhospital mortality after percutaneous coronary intervention and could be used for risk stratification [14]. Moreover, a meta-analysis and another study revealed that AG may be a good choice to assess the prognosis of critically ill patients especially for those in areas with inadequate medical resources $[15,28]$. Similarly, our data suggested that AG was associated with inhospital allcause mortality of CCU patients independently, and maybe the adverse effects of increased AG on coronary artery disease and critically ill patients contribute to this result. Moreover, AG contributed to the diagnosis of acute kidney injury [8, 27]. Similarly, we found that as AG quartiles increased, incidence of acute kidney injury increased significantly. SOFA and SAPSII were good scoring system for predicting the prognosis of critically ill patients. In this study, we found that as AG increased, SOFA and SAPSII increased significantly, and this phenomenon may also explain higher inhospital mortality in patients with higher AG. Moreover, length of CCU and hospital stay prolonged significantly in higher AG quartiles, which will bring greater psychological, physical, and financial burden to patients, so more attention to AG in CCU patients may be needed.

In coronary artery disease, acute myocardial infarction, third-degree atrioventricular block, congestive heart failure, primary cardiomyopathy, valve disease, ventricular arrhythmias, endocarditis, cardiogenic shock, and atrial fibrillation subgroups, we can all come to the same conclusion that as AG increased, inhospital mortality increased. All above 


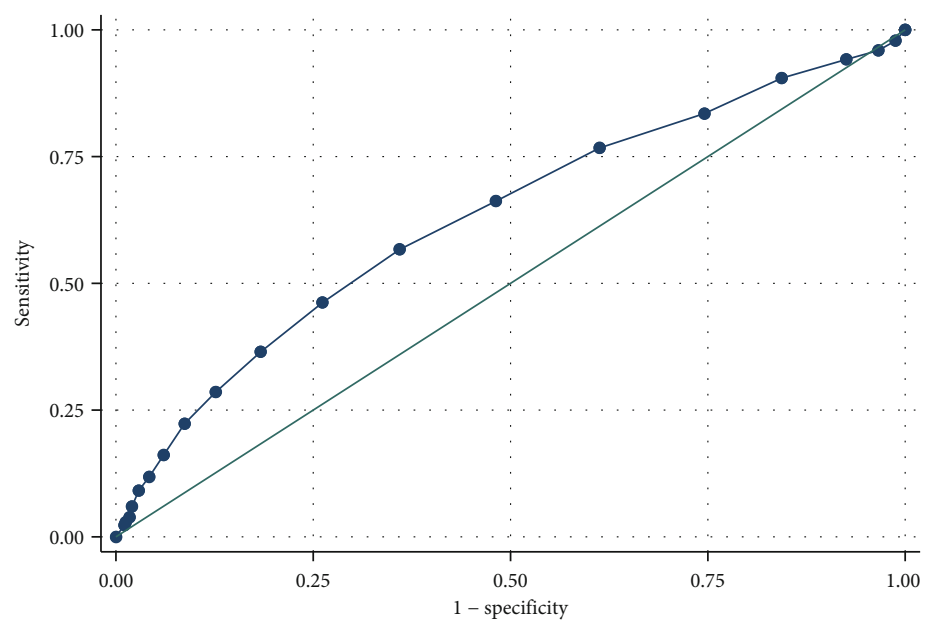

Area under ROC curve $=0.6291$

(a)

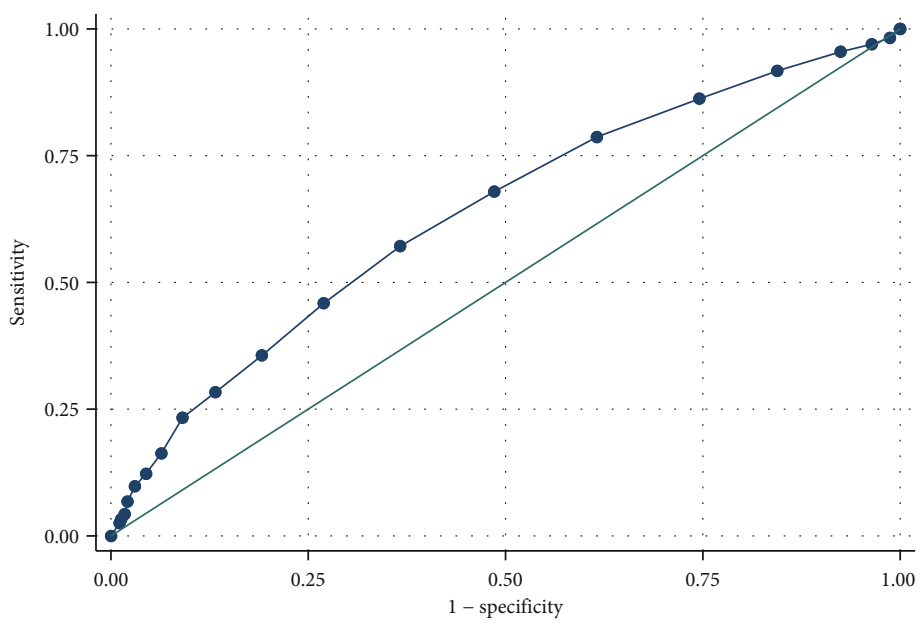

Area under ROC curve $=0.6355$

(b)

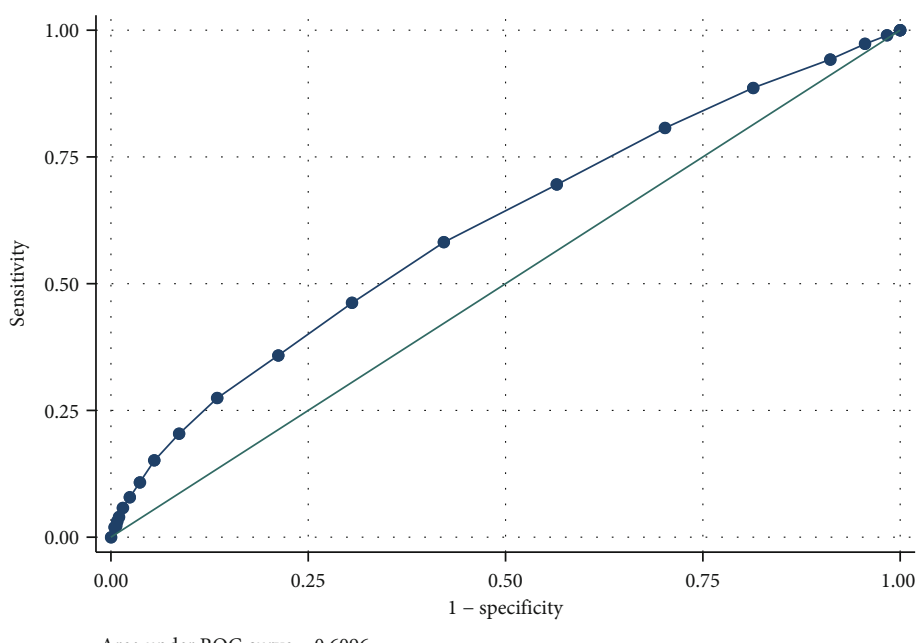

(c)

FIGURE 3: ROC curves of AG for prediction of inhospital all-cause mortality (a), CCU all-cause mortality (b), and acute kidney injury (c). Abbreviation: AG: anion gap; CCU: coronary care unit. 
diseases almost covered most diseases of CCU. This result provided a strong support for us to use AG as a clinical indicator in CCU to predict prognosis. In hypercholesterolemia, congestive heart failure, chronic lung disease, respiratory failure, oral anticoagulants, Beta-blocks, ACEI/ARB, and vasopressin subgroups, the interactions were proved to be significant. Further research is needed to clarify the reasons.

\section{Limitation}

This study was a single retrospective study, and inevitable bias may affect the authenticity of the results. Moreover, the bulk of AG is largely determined by anions attached to circulation protein $[29,30]$, and as albumin decreases, so does AG [31, 32]. But due to the loss of albumin data, we did not include the albumin data in this study. Apart from the retrospective model, the main bias of this study was the lack of albumin values for a correct AG adjustment. In general, the more key variables a model contains, the more accurate its predictions will be. But constrained by public databases, a lot of information that may affect the model was not collected, like smoking and drinking alcohol. In addition to this, other important information was also not collected such as specific cause of death, cardiac function, and left ventricular ejection fraction. In order to verify the conclusion, prospective case-control study may be needed.

\section{Conclusion}

AG was an independent risk factor of inhospital all-cause mortality and was associated with adverse clinical outcomes in CCU patients. But more prospective case-control data are needed to confirm AG's role as a clinical indicator in $\mathrm{CCU}$ to predict prognosis.

\section{Data Availability}

All data used in this analysis were from an openly available critical care database named MIMIC-III. Protecting Human Research Participant exam was passed to gain access to MIMIC-III database, and our certificate number is 9027152 .

\section{Ethical Approval}

Ethics approval of MIMIC-III database was obtained by the institutional review boards at Beth Israel Deaconess Medical Center and Massachusetts Institute of Technology.

\section{Conflicts of Interest}

The authors declare that they have no conflicts of interest.

\section{Acknowledgments}

Thanks to all co-authors for the contributions to data collection and processing.

\section{References}

[1] S. Geneva and World Health Organization, Global Status Report on Noncommunicable Diseases 2010, World Health Organization, 2011.

[2] G. A. Roth, D. Abate, K. H. Abate et al., "Global, regional, and national age-sex-specific mortality for 282 causes of death in 195 countries and territories, 1980-2017: a systematic analysis for the Global Burden of Disease Study 2017," Lancet, vol. 392, no. 10159, pp. 1736-1788, 2018.

[3] W. B. Fye, "Resuscitating a circulation abstract to celebrate the 50th anniversary of the coronary care unit concept," Circulation, vol. 124, no. 17, pp. 1886-1893, 2011.

[4] D. G. Julian, "The history of coronary care units," British Heart Journal, vol. 57, no. 6, pp. 497-502, 1987.

[5] T. Killip 3rd and J. T. Kimball, "Treatment of myocardial infarction in a coronary care unit," The American Journal of Cardiology, vol. 20, no. 4, pp. 457-464, 1967.

[6] R. L. MacMillan and K. W. Brown, "Comparison of the effects of treatment of acute myocardial infarction in a coronary unit and on a general medical ward," Canadian Medical Association Journal, vol. 105, no. 10, pp. 1037-1040, 1971.

[7] J. Loughran, T. Puthawala, B. S. Sutton, L. E. Brown, P. J. Pronovost, and A. P. DeFilippis, "The cardiovascular intensive care unit-an evolving model for health care delivery," Journal of Intensive Care Medicine, vol. 32, no. 2, pp. 116123, 2016.

[8] J. A. Kraut and N. E. Madias, "Serum anion gap: its uses and limitations in clinical medicine," Clinical journal of the American Society of Nephrology, vol. 2, no. 1, pp. 162-174, 2006.

[9] J. A. Kraut and G. T. Nagami, "The serum anion gap in the evaluation of acid-base disorders: what are its limitations and can its effectiveness be improved?," Clinical Journal of the American Society of Nephrology : CJASN, vol. 8, no. 11, pp. 2018-2024, 2013.

[10] S. W. Yang, Y. J. Zhou, Y. X. Zhao et al., "The serum anion gap is associated with disease severity and all-cause mortality in coronary artery disease," Journal of geriatric cardiology : JGC, vol. 14, no. 6, pp. 392-400, 2017.

[11] Q. Chen, Q. Chen, L. Li et al., "Serum anion gap on admission predicts intensive care unit mortality in patients with aortic aneurysm," Experimental and Therapeutic Medicine, vol. 16, no. 3, pp. 1766-1777, 2018.

[12] A. Sahu, H. A. Cooper, and J. A. Panza, "The initial anion gap is a predictor of mortality in acute myocardial infarction," Coronary Artery Disease, vol. 17, no. 5, pp. 409-412, 2006.

[13] P. Attanà, C. Lazzeri, M. Chiostri, C. Picariello, G. F. Gensini, and S. Valente, "Strong-ion gap approach in patients with cardiogenic shock following ST-elevation myocardial infarction," Acute Cardiac Care, vol. 15, no. 3, pp. 58-62, 2013.

[14] C. Lazzeri, S. Valente, M. Chiostri, C. Picariello, and G. F. Gensini, "Evaluation of acid-base balance in ST-elevation myocardial infarction in the early phase: a prognostic tool?," Coronary Artery Disease, vol. 21, no. 5, pp. 266-272, 2010.

[15] S. A. Glasmacher and W. Stones, "Anion gap as a prognostic tool for risk stratification in critically ill patients - a systematic review and meta-analysis," BMC Anesthesiology, vol. 16, no. 1, p. $68,2016$.

[16] E. N. Taylor, J. P. Forman, and W. R. Farwell, "Serum anion gap and blood pressure in the national health and nutrition 
examination survey," Hypertension, vol. 50, no. 2, pp. 320-324, 2007.

[17] W. R. Farwell and E. N. Taylor, "Serum bicarbonate, anion gap and insulin resistance in the National Health and Nutrition Examination Survey," Diabetic Medicine : a Journal of the British Diabetic Association, vol. 25, no. 7, pp. 798-804, 2008.

[18] M. K. Abramowitz, T. H. Hostetter, and M. L. Melamed, "Lower serum bicarbonate and a higher anion gap are associated with lower cardiorespiratory fitness in young adults," Kidney International, vol. 81, no. 10, pp. 1033-1042, 2012.

[19] M. Park, S. J. Jung, S. Yoon, J. M. Yun, and H. J. Yoon, “Association between the markers of metabolic acid load and higher all-cause and cardiovascular mortality in a general population with preserved renal function," Hypertension Research : official Journal of the Japanese Society of Hypertension, vol. 38, no. 6, pp. 433-438, 2015.

[20] A. E. Johnson, T. J. Pollard, L. Shen et al., "MIMIC-III, a freely accessible critical care database," Scientific Data, vol. 3, no. 1, p. $160035,2016$.

[21] H. Quan, B. Li, L. Duncan Saunders et al., "Assessing validity of ICD-9-CM and ICD-10 administrative data in recording clinical conditions in a unique dually coded database," Health Services Research, vol. 43, no. 4, pp. 1424-1441, 2008.

[22] A. N. Mehta, J. B. Emmett, and M. Emmett, "GOLD MARK: an anion gap mnemonic for the 21st century," Lancet, vol. 372, no. 9642 , p. 892, 2008.

[23] J. L. Vincent, R. Moreno, J. Takala et al., "The SOFA (sepsis-related organ failure assessment) score to describe organ dysfunction/failure. On behalf of the Working Group on Sepsis-Related Problems of the European Society of Intensive Care Medicine," Intensive Care Medicine, vol. 22, no. 7, pp. 707-710, 1996.

[24] J. R. Le Gall, S. Lemeshow, and F. Saulnier, "A new simplified acute physiology score (SAPS II) based on a European/North American multicenter study," Journal of the American Medical Association, vol. 270, no. 24, pp. 2957-2963, 1993.

[25] R. L. Mehta, J. A. Kellum, S. V. Shah et al., “Acute Kidney Injury Network: report of an initiative to improve outcomes in acute kidney injury," Critical Care, vol. 11, no. 2, p. R31, 2007.

[26] L. L. Hamm, N. Nakhoul, and K. S. Hering-Smith, "Acid-base homeostasis," Clinical Journal of the American Society of Nephrology: CJASN, vol. 10, no. 12, pp. 2232-2242, 2015.

[27] I. Co and K. Gunnerson, "Emergency Department Management of Acute Kidney Injury, Electrolyte Abnormalities, and Renal Replacement Therapy in the Critically Ill," Emergency Medicine Clinics of North America, vol. 37, no. 3, pp. 459471, 2019.

[28] C. M. Zheng, W. C. Liu, J. Q. Zheng et al., "Metabolic acidosis and strong ion gap in critically ill patients with acute kidney injury," BioMed Research International, vol. 2014, Article ID 819528, 8 pages, 2014.

[29] H. E. Corey, "The anion gap (AG): studies in the nephrotic syndrome and diabetic ketoacidosis (DKA)," The Journal of Laboratory and Clinical Medicine, vol. 147, no. 3, pp. 121$125,2006$.

[30] M. Feldman, N. Soni, and B. Dickson, "Influence of hypoalbuminemia or hyperalbuminemia on the serum anion gap," The Journal of Laboratory and Clinical Medicine, vol. 146, no. 6, pp. 317-320, 2005.
[31] J. Figge, A. Jabor, A. Kazda, and V. Fencl, "Anion gap and hypoalbuminemia," Critical Care Medicine, vol. 26, no. 11, pp. 1807-1810, 1998.

[32] C. P. Carvounis and D. A. Feinfeld, "A simple estimate of the effect of the serum albumin level on the anion gap," American Journal of Nephrology, vol. 20, no. 5, pp. 369-372, 2000. 